\title{
Ratio Asymptotics for Orthogonal Rational Functions on the Interval $[-1,1]$
}

\author{
J. Van Deun and A. Bultheel \\ Department of Computer Science, K.U.Leuven, Belgium \\ E-mail: \{joris.vandeun-adhemar.bultheel\}@cs.kuleuven.ac.be
}

\begin{abstract}
Let $\left\{\alpha_{1}, \alpha_{2}, \ldots\right\}$ be a sequence of real numbers outside the interval $[-1,1]$ and $\mu$ a positive bounded Borel measure on this interval. We introduce rational functions $\varphi_{n}(x)$ with poles $\left\{\alpha_{1}, \ldots, \alpha_{n}\right\}$ orthogonal on $[-1,1]$ and establish some ratio asymptotics for these orthogonal rational functions, i.e. we discuss the convergence of $\varphi_{n+1}(x) / \varphi_{n}(x)$ as $n$ tends to infinity under certain assumptions on the measure and the location of the poles. From this we derive asymptotic formulas for the recurrence coefficients in the three term recurrence relation satisfied by the orthonormal functions.
\end{abstract}

\section{Introduction}

Ratio asymptotics for orthogonal polynomials on the interval $[-1,1]$ have been studied in several books and papers. Most results were obtained relating these polynomials to polynomials orthogonal on the unit circle in the complex plane using the Joukowski transform $x=\frac{1}{2}\left(z+z^{-1}\right)$ which maps the unit circle to the interval $[-1,1]$. In this way Szegö [9] obtained convergence results for weights satisfying Szegö's condition, and the general case was treated by Rakhmanov $[6,7]$. See also $[4,5]$ for results about polynomials orthogonal with respect to varying measures.

Orthogonal rational functions are a generalization of orthogonal polynomials, in such a way that we recover the polynomial situation if all poles are at infinity. Asymptotics for rational functions orthogonal on the unit circle 
(or, using a Cayley transform, on the extended real line) were studied in [1], but the case of a finite interval has thus far not been treated. In this paper it is our aim to derive convergence results for orthogonal rational functions on the interval $[-1,1]$, using a relation between rational functions on the unit circle and the interval $[-1,1]$ as described in [10].

Just as in the polynomial case orthogonal rational functions satisfy a three term recurrence relation. Asymptotics for the recurrence coefficients can be derived in a very natural way from the ratio asymptotics for the orthogonal functions, as in [6].

In the next sections we introduce the spaces of rational functions we are dealing with and we discuss the recurrence relation. We will need several lemmas before we can state our main result about the convergence of the ratio of orthogonal rational functions in section 6 . Using this result it is not difficult to derive asymptotics for the recurrence coefficients.

\section{Preliminaries}

The complex plane is denoted by $\mathbb{C}$, the Riemann sphere by $\hat{\mathbb{C}}=\mathbb{C} \cup\{\infty\}$, the real line by $\mathbb{R}$ and the extended real line by $\hat{\mathbb{R}}=\mathbb{R} \cup\{\infty\}$. For the unit circle, its interior and its exterior we introduce the following notation:

$$
\mathbb{T}=\{z:|z|=1\}, \quad \mathbb{D}=\{z:|z|<1\}, \quad \mathbb{E}=\{z:|z|>1\}
$$

We will also use $I=[-1,1], \mathbb{R}^{I}=\hat{\mathbb{R}} \backslash I$ and $\mathbb{C}^{I}=\hat{\mathbb{C}} \backslash I$. Given positive bounded Borel measures $\mu$ on $I$ and $\nu$ on $[0,2 \pi]$ respectively, the inner product is defined as

$$
\begin{aligned}
\langle f, g\rangle & =\int_{-1}^{1} f(z) \overline{g(z)} d \mu(z), \quad \text { on } I, \\
& =\int_{0}^{2 \pi} f\left(e^{\mathbf{i} \theta} \overline{g\left(e^{\mathbf{i} \theta}\right)} d \nu(\theta), \quad \text { on } \mathbb{T}\right.
\end{aligned}
$$

In [1] the measure is assumed to be normalized, i.e. $\langle 1,1\rangle=1$. The convergence results from section 4 were proved under that assumption, but it is not difficult to see that they remain valid in the case of a nonnormalized measure. Therefore in what follows we will drop the normalization assumption. The convergence results for the unit circle in section 4 depend on the divergence to zero of the Blaschke products $B_{n}(z)$ defined in this section. 
The Blaschke factors as defined in [1] differ from the ones we define further on by a factor $-\bar{\beta}_{n} /\left|\beta_{n}\right|$. These unimodular constants are needed to ensure the convergence of the Blaschke products, but if they diverge to zero, they diverge with or without these constant factors. Since this type of divergent Blaschke products are considered in this paper, the presence of these constants is irrelevant for our results. We have used the definitions from [10].

Now we are ready to introduce the space of rational functions with real poles in $\mathbb{R}^{I}$. Let a sequence $\left\{\alpha_{1}, \alpha_{2}, \ldots\right\} \subset \mathbb{R}^{I}$ and a positive bounded Borel measure $\mu$ with $\operatorname{supp}(\mu) \subset I$ an infinite set be given (where $\operatorname{supp}(\mu)$ means the support of the measure, i.e. the smallest closed set whose complement has $\mu$ measure zero). Define factors

$$
Z_{n}(z)=\frac{-\alpha_{n} z}{z-\alpha_{n}}
$$

and basis functions

$$
b_{n}(z)=b_{n-1}(z) Z_{n}(z), \quad b_{0}(z)=1 .
$$

Then we define the space of rational functions with poles in $\left\{\alpha_{1}, \ldots, \alpha_{n}\right\}$ as

$$
\mathcal{L}_{n}=\operatorname{span}\left\{b_{0}, \ldots, b_{n}\right\} .
$$

After orthonormalization of the basis $\left\{b_{0}, \ldots, b_{n}\right\}$ with respect to $\mu$ we obtain orthogonal rational functions $\left\{\varphi_{0}, \ldots, \varphi_{n}\right\}$ where we choose the leading coefficient $k_{n}$ in the expansion $\varphi_{n}(z)=k_{n} b_{n}(z)+\ldots$ such that it satisfies $k_{n}>0$.

The orthogonal rational functions on the unit circle are defined similarly. Let a sequence of complex numbers $\left\{\beta_{1}, \beta_{2}, \ldots\right\} \subset \mathbb{D}$ and a positive bounded Borel measure $\nu$ on $\mathbb{T}$ be given (again assume $\operatorname{supp}(\nu)$ is an infinite set). Define the Blaschke factors

$$
\zeta_{n}(z)=\frac{z-\beta_{n}}{1-\bar{\beta}_{n} z}
$$

and Blaschke products

$$
B_{n}(z)=\zeta_{n}(z) B_{n-1}(z), \quad B_{0}(z)=1
$$

and the space of rational functions on the unit circle,

$$
\stackrel{\circ}{\mathcal{L}}_{n}=\operatorname{span}\left\{B_{0}, \ldots, B_{n}\right\} .
$$


Note that the poles $\left\{1 / \bar{\beta}_{1}, \ldots, 1 / \bar{\beta}_{n}\right\}$ of a function $f \in \stackrel{\circ}{\mathcal{L}}_{n}$ are in $\mathbb{E}$. Orthonormalizing the basis $\left\{B_{0}, \ldots, B_{n}\right\}$ we obtain the orthogonal functions $\left\{\phi_{0}, \ldots, \phi_{n}\right\}$ where again we choose the leading coefficient $\kappa_{n}$ to be positive.

Now define the para-hermitian conjugate of a function $f(z)$ as

$$
f_{*}(z)=\overline{f(1 / \bar{z})}
$$

and the superstar transform of $\phi_{n}$ as

$$
\phi_{n}^{*}(z)=B_{n}(z) \phi_{n *}(z) .
$$

Note that $\phi_{n}^{*}$ is a function in $\stackrel{\circ}{\mathcal{L}}_{n}$.

\section{Three term recurrence}

As in the polynomial case, orthogonal rational functions on (an interval of) the real line satisfy a three term recurrence relation. We shall call the orthogonal rational function $\varphi_{n}$ singular if $p_{n}\left(\alpha_{n-1}\right)=0$, where $p_{n}$ is the numerator polynomial of $\varphi_{n}$, and regular otherwise. If we put by convention $\alpha_{-1}=\alpha_{0}=\infty$ then it can be shown [1] that the orthonormal functions $\varphi_{n}$ satisfy the following three term recurrence relation with the initial conditions $\varphi_{-1}(z)=0, \varphi_{0}(z)=1$ iff both $\varphi_{n-1}$ and $\varphi_{n}$ are regular,

$$
\varphi_{n}(z)=\left(E_{n} Z_{n}(z)+B_{n} \frac{Z_{n}(z)}{Z_{n-1}(z)}\right) \varphi_{n-1}(z)-\frac{E_{n}}{E_{n-1}} \frac{Z_{n}(z)}{Z_{n-2}(z)} \varphi_{n-2}(z) .
$$

In the case of orthogonality on $I$ and poles in $\mathbb{R}^{I}$ the regularity conditions are always satisfied. This follows from the following theorem, which can be found in [2].

Theorem 3.1. Let $\varphi_{n}$ be an orthogonal rational function on the interval $[-1,1]$ with poles outside this interval. Then the zeros of $\varphi_{n}$ are simple and contained in the open interval $(-1,1)$.

In [2] the measure was assumed to be absolutely continuous with respect to the Lebesgue measure, but the theorem still holds for arbitrary measures if the support of the measure is an infinite set (the proof proceeds along the same lines as the classical proof on the location of the zeros of orthogonal polynomials, see e.g. [9, p.44]). Having established the regularity of the system $\left\{\varphi_{n}\right\}$ we know that the recurrence relation (1) holds for all $n \geq$ 1. It thus makes sense to study the asymptotic behavior of the recurrence coefficients $E_{n}$ and $B_{n}$ as $n$ tends to infinity. 


\section{Convergence results for the unit circle}

In this section we will recall some convergence results about orthogonal rational functions on the unit circle with poles in $\mathbb{E}$, as stated in [1]. In the following, locally uniform convergence in a region $\Omega$ will mean uniform convergence on compact subsets of $\Omega$.

With the notation of section 2 , let $\nu^{\prime}$ be the Radon-Nikodym derivative of the measure $\nu$ with respect to the Lebesgue measure (i.e. the density of the absolutely continuous part of $\nu)$. Szegö's condition $\log \nu^{\prime} \in L^{1}[0,2 \pi]$ means

$$
\int_{0}^{2 \pi} \log \nu^{\prime}(\theta) d \theta>-\infty
$$

Next we introduce the Riesz-Herglotz kernel

$$
D(t, z)=\frac{t+z}{t-z}
$$

and its real part, the Poisson kernel

$$
P(t, z)=\Re D(t, z)=\frac{1-|z|^{2}}{|t-z|^{2}}
$$

where $t \in \mathbb{T}$ and $z \in \mathbb{D}$. If Szegö's condition is satisfied, we can define the normalized Szegö function $S_{w}(z)$ as

$$
S_{w}(z)=\exp \left\{-\frac{1}{4 \pi} \int_{0}^{2 \pi} D\left(e^{\mathbf{i} \theta}, z\right) \log \left[\frac{\nu^{\prime}(\theta)}{P\left(e^{\mathbf{i} \theta}, w\right)}\right] d \theta\right\}, \quad w \in \mathbb{D} .
$$

We are now in a position to formulate the convergence results that we will need for our main theorem. They can be found in chapter 9 of [1].

Theorem 4.1. Let $\left\{\beta_{1}, \beta_{2}, \ldots\right\} \subset \mathbb{D}$ be a sequence which is compactly included in $\mathbb{D}$ and $\left\{\phi_{0}, \phi_{1}, \ldots\right\}$ the orthonormal functions associated with the basisfunctions $\left\{B_{0}, B_{1}, \ldots\right\}$ as defined in section 2, and let $\nu$ be a positive bounded Borel measure on $\mathbb{T}$ satisfying Szego"'s condition (2). Then

$$
\lim _{n \rightarrow \infty} \phi_{n}(z)=0
$$

locally uniformly in $\mathbb{D}$, the sequence $\left\{\kappa_{n}\right\}$ is bounded and the sequence $\left\{\phi_{n}^{*}(z)\right\}$ is locally uniformly bounded in $\mathbb{D}$. If furthermore the sequence $\left\{\beta_{1}, \beta_{2}, \ldots\right\}$ has a convergent subsequence $\lim _{s \rightarrow \infty} \beta_{n(s)}=\beta$ then

$$
\lim _{s \rightarrow \infty} \phi_{n(s)}^{*}(z)=S_{\beta}(z)
$$


locally uniformly in $\mathbb{D}$ and

$$
\lim _{s \rightarrow \infty} \kappa_{n(s)}=S_{\beta}(\beta)
$$

Note that the last equality follows from $\kappa_{n}=\phi_{n}^{*}\left(\beta_{n}\right)$ and the fact that convergence is uniform. The condition that the sequence $\left\{\beta_{1}, \beta_{2}, \ldots\right\}$ is compactly included in $\mathbb{D}$ implies that it is bounded away from the boundary $\mathbb{T}$.

\section{Relating the unit circle to the interval}

In this section we will use $x$ as the independent variable for the orthonormal rational functions $\varphi_{n}(x)$ on $I$ and $z$ for the functions $\phi_{n}(z)$ orthogonal on the unit circle. They can be related to each other in more or less the same way orthogonal polynomials on $I$ can be related to orthogonal polynomials on the unit circle, see [9]. The relations in this section were derived in [10].

We will denote the Joukowski transform $x=\frac{1}{2}\left(z+z^{-1}\right)$ by $x=J(z)$, mapping the open unit disc $\mathbb{D}$ to the cut Riemann sphere $\mathbb{C}^{I}$ and the unit circle $\mathbb{T}$ to the interval $I$. The inverse mapping is denoted by $z=J^{-1}(x)$ and is chosen so that $z \in \mathbb{D}$ if $x \in \mathbb{C}^{I}$. To the sequence $\left\{\alpha_{1}, \alpha_{2}, \ldots\right\} \subset \mathbb{R}^{I}$ we associate a sequence $\left\{\beta_{1}, \beta_{2}, \ldots\right\} \subset I$ such that $\beta_{k}=J^{-1}\left(\alpha_{k}\right)$, and a sequence $\left\{\tilde{\beta}_{1}, \tilde{\beta}_{2}, \ldots\right\}$ such that $\tilde{\beta}_{2 k}=\tilde{\beta}_{2 k-1}=\beta_{k}$. The corresponding Blaschke products and orthogonal functions are denoted by a tilde. Obviously $\tilde{B}_{2 k}=\left(B_{k}\right)^{2}$. The fact that $\left\{\alpha_{n}\right\} \subset \mathbb{R}^{I}$ implies that $\left\{\beta_{n}\right\}$ is actually in the open interval $(-1,1)$ and thus in $\mathbb{D}$.

Next define the measure ${ }^{1} \nu$ on $\mathbb{T}$ as

$$
\nu(E)=\mu(\{\cos \theta, \theta \in E \cap[0, \pi)\})+\mu(\{\cos \theta, \theta \in E \cap[\pi, 2 \pi)\}) .
$$

This is sometimes written $\nu(E)=\int_{E}|d \mu(\cos \theta)|$, but we prefer the less ambiguous notation. Using the Lebesgue decomposition of $\mu$ and the changeof-variables theorem (see e.g. [8, p. 153]) it is not difficult to see that

$$
\nu^{\prime}(\theta)=\mu^{\prime}(\cos \theta)|\sin \theta| .
$$

Then if $\left\{\tilde{\phi}_{n}\right\}$ is the orthonormal set associated with the sequence $\left\{\tilde{\beta}_{1}, \tilde{\beta}_{2}, \ldots\right\}$ and the measure $\nu$, we have the following theorem.

\footnotetext{
${ }^{1}$ In [10] the measure $\mu$ was assumed to be absolutely continuous, but this can easily be extended to arbitrary positive Borel measures whose support is an infinite set. See also $[3$, p.190] for the polynomial case
} 
Theorem 5.1. Let $\left\{\varphi_{n}\right\}$ be a set of orthonormal rational functions on I and $\left\{\tilde{\phi}_{n}\right\}$ the corresponding set of functions orthogonal on $\mathbb{T}$ with poles and measure as defined above, then they are related by

$$
\varphi_{n}(x)=(2 \pi)^{-\frac{1}{2}}\left\{1+\frac{\tilde{\phi}_{2 n}\left(\beta_{n}\right)}{\tilde{\kappa}_{2 n}}\right\}^{-\frac{1}{2}}\left\{B_{n *}(z) \tilde{\phi}_{2 n}(z)+B_{n} \tilde{\phi}_{2 n *}(z)\right\}
$$

where $x=J(z)$.

Note that we have to double the multiplicity of every pole to obtain these results.

\section{$6 \quad$ Ratio asymptotics}

With the aid of theorems 4.1 and 5.1 we are able to prove our main results about the convergence of the ratio of orthogonal rational functions on $I$. Of course more restrictive conditions on the location of the poles lead to more specific convergence results. In the sequel we will use the concept of aymptotic periodicity, which we define as follows: a sequence $\left\{\alpha_{1}, \alpha_{2}, \ldots\right\}$ is asymptotically periodic with period $m$ if there exists a periodic sequence $\left\{\alpha_{1}^{0}, \alpha_{2}^{0}, \ldots\right\}$

$$
\alpha_{n+m}^{0}=\alpha_{n}^{0}, \quad n=1,2, \ldots
$$

such that

$$
\lim _{n \rightarrow \infty}\left|\alpha_{n}-\alpha_{n}^{0}\right|=0 .
$$

Now we can state our first result. The following theorem corresponds to the special case $m=1$ in theorem 6.4, but since the expressions simplify considerably, we state it separately.

Theorem 6.1. Let a sequence of poles $A=\left\{\alpha_{1}, \alpha_{2}, \ldots\right\} \subset \mathbb{R}^{I}$ be given such that $\lim _{n \rightarrow \infty} \alpha_{n}=\alpha \in \mathbb{R}^{I}$, and let $\mu$ be a positive bounded Borel measure with $\operatorname{supp}(\mu) \subset I$ an infinite set, which satisfies the condition

$$
\int_{-1}^{1} \frac{\log \mu^{\prime}(x)}{\sqrt{1-x^{2}}} d x>-\infty .
$$


If $\left\{\varphi_{n}\right\}$ are the orthonormal rational functions on $I$ associated with $A$ and $\mu$, then the ratio $\varphi_{n+1}(x) / \varphi_{n}(x)$ converges to a meromorphic function $F_{\alpha}(x)$. Convergence is locally uniform in $\mathbb{C}^{I} \backslash\{\alpha\}$ and pointwise in $\mathbb{C}^{I}$. If we define $\beta \in I$ and $z \in \mathbb{D}$ such that $\alpha=J(\beta)$ and $x=J(z)$, then

$$
F_{\alpha}(x)=\frac{1}{\zeta_{\beta}(z)} \quad \text { and } \quad\left|F_{\alpha}(x)\right|>1, x \in \mathbb{C}^{I},
$$

where $\zeta_{\beta}(z)=(z-\beta) /(1-\beta z)$.

If $|\alpha|<\infty$ then $F_{\alpha}(x)$ has a single pole at $x=\alpha$ and a Laurent series expansion in a neighbourhood of $\alpha$

$$
F_{\alpha}(x)=\frac{2\left(1-\alpha^{2}\right)}{x-\alpha}-2 \alpha+O(x-\alpha) .
$$

If $|\alpha|=\infty$ then

$$
F_{\infty}(x)=x+\sqrt{x^{2}-1}
$$

where the branch of the square root is chosen so that $\left|F_{\infty}(x)\right|>1, x \in \mathbb{C}^{I}$.

Proof. Define a measure $\nu$ on $\mathbb{T}$ by (3) and then use theorem 5.1 to write

$$
\frac{\varphi_{n+1}(x)}{\varphi_{n}(x)}=\sqrt{\frac{1+\frac{\tilde{\phi}_{2 n}\left(\beta_{n}\right)}{\tilde{\kappa}_{2 n}}}{1+\frac{\tilde{\phi}_{2 n+2}\left(\beta_{n+1}\right)}{\tilde{\kappa}_{2 n+2}}}} \frac{B_{(n+1) *}(z) \tilde{\phi}_{2 n+2}(z)+B_{n+1}(z) \tilde{\phi}_{(2 n+2) *}(z)}{B_{n *}(z) \tilde{\phi}_{2 n}(z)+B_{n}(z) \tilde{\phi}_{2 n *}(z)} .
$$

With the definitions given in the previous sections this expression is easily transformed into

$$
\frac{\varphi_{n+1}(x)}{\varphi_{n}(x)}=\sqrt{\frac{1+\frac{\tilde{\phi}_{2 n}\left(\beta_{n}\right)}{\tilde{\kappa}_{2 n}}}{1+\frac{\tilde{\phi}_{2 n+2}\left(\beta_{n+1}\right)}{\tilde{\kappa}_{2 n+2}}}} \frac{1}{\zeta_{n+1}(z)} \frac{\tilde{\phi}_{2 n+2}(z)+\tilde{\phi}_{2 n+2}^{*}(z)}{\tilde{\phi}_{2 n}(z)+\tilde{\phi}_{2 n}^{*}(z)} .
$$

It follows from theorem 5.2.1. in [1] that the zeros of $\tilde{\phi}_{2 n}(z)+\tilde{\phi}_{2 n}^{*}(z)$ are all on $\mathbb{T}$. Now take a compact subset $\Omega$ of $\mathbb{D} \backslash \beta$. The fact that $\lim _{n \rightarrow \infty} \alpha_{n}=\alpha$ implies that $\lim _{n \rightarrow \infty} \beta_{n}=\beta$ and this means that there exists an $N$ such that $\zeta_{n}(z)$ has no zeros on $\Omega$ for $n>N$. Furthermore it follows from theorem 4.1 and the definition of the normalized Szegö function $S_{\beta}(z)$ that the sequence $\left\{\tilde{\kappa}_{2 n}\right\}$ is bounded away from zero. Also, formulas (3)-(5) show that

$$
\int_{0}^{2 \pi} \log \nu^{\prime}(\theta) d \theta>-\infty
$$


All this combined with theorem 4.1 then shows that

$$
\lim _{n \rightarrow \infty} \frac{\varphi_{n+1}(x)}{\varphi_{n}(x)}=F_{\alpha}(x)=\frac{1}{\zeta_{\beta}(z)}
$$

locally uniformly in $\mathbb{C}^{I} \backslash\{\alpha\}$ and pointwise in $\mathbb{C}^{I}$, proving (6). This is obviously a meromorphic function which has a single pole at $z=\beta$ and which is greater than 1 in modulus.

If $\beta=0$ then $|\alpha|=\infty$ and $\zeta_{0}(z)=z$ so $F_{\infty}(x)=1 / J^{-1}(x)$ which yields formula (8).

If $\beta \neq 0$ then $|\alpha|<\infty$ and we may expand $F_{\alpha}(x)$ in a Laurent series in a neighbourhood of $\alpha$

$$
F_{\alpha}(x)=\frac{a_{-1}}{x-\alpha}+a_{0}+a_{1}(x-\alpha)+\ldots
$$

The coefficients $\left\{a_{k}\right\}$ are calculated from

$$
a_{k}=\frac{1}{(k+1) !} \lim _{x \rightarrow \alpha} \frac{d^{k+1}}{d x^{k+1}}\left\{F_{\alpha}(x)(x-\alpha)\right\}
$$

using $F_{\alpha}(x)(x-\alpha)=\frac{1}{2}(1-\beta z)\left(1-\frac{1}{\beta z}\right)$ and the fact that $x=J(z)$. Some algebra then yields $(7)$.

An explicit formula for $F_{\alpha}(x)$ can be derived using the relations $\alpha=J(\beta)$ and $x=J(z)$. The result is stated in the following corollary.

Corollary 6.2. The function $F_{\alpha}(x)$ as defined in theorem 6.1 equals

$$
F_{\alpha}(x)=\frac{1-\alpha x}{x-\alpha}-\frac{\sqrt{\left(x^{2}-1\right)\left(\alpha^{2}-1\right)}}{x-\alpha}
$$

where the branch of the square root is chosen so that $\left|F_{\alpha}(x)\right|>1$ for $x \in \mathbb{C}^{I}$.

Proof. This is a matter of simple algebra.

Note that $\lim _{\alpha \rightarrow \infty} F_{\alpha}(x)=F_{\infty}(x)$ as defined through formula (8). The function $F_{\infty}(x)$ is the same limit function as in the polynomial case, see e.g. [6]. This also follows from the fact that we recover the polynomial situation if all poles are at infinity.

We now turn to the more general case of an asymptotically periodic pole sequence. The following lemma will be useful. 
Lemma 6.3. Let $S_{w}(z)$ denote the normalized Szegö function as defined in section 4. Then for $u, v \in \mathbb{D}$ we have

$$
\frac{S_{u}(z)}{S_{v}(z)}=S_{u, v}(z)=\left\{\frac{1-|u|^{2}}{1-|v|^{2}}\right\}^{\frac{1}{2}} \exp \left\{-\frac{z}{\pi} \int_{0}^{2 \pi} \frac{\log \left|\frac{e^{\mathrm{i} \theta}-u}{e^{\mathrm{i} \theta}-v}\right|}{e^{\mathrm{i} \theta}-z} d \theta\right\}
$$

Proof. This follows from the definition of $S_{w}(z)$ and $D(t, z)$, using

$$
\frac{1}{2 \pi} \int_{0}^{2 \pi} D\left(e^{\mathbf{i} \theta}, z\right) d \theta=1, \quad z \in \mathbb{D}
$$

and

$$
\int_{0}^{2 \pi} \log \left|e^{\mathrm{i} \theta}-w\right| d \theta=0, \quad w \in \mathbb{D}
$$

The last equality can be proved using Cauchy's theorem, see e.g. [8, p.307] for a similar integral.

We state our second convergence theorem.

Theorem 6.4. Assume the sequence $A=\left\{\alpha_{1}, \alpha_{2}, \ldots\right\} \subset \mathbb{R}^{I}$ is asymptotically m-periodic with limiting values $\left\{\alpha_{i}^{0}\right\}_{i=1}^{m} \subset \mathbb{R}^{I}$ and assume the measure $\mu$ satisfies the conditions of theorem 6.1. If $\left\{\varphi_{n}\right\}$ are the orthonormal rational functions on $I$ associated with $A$ and $\mu$, then we have with the notation introduced above

$$
\lim _{n \rightarrow \infty} \frac{\varphi_{n m+i}(x)}{\varphi_{n m+i-1}(x)}=\frac{S_{\beta_{i}^{0}, \beta_{i-1}^{0}}(z)}{\zeta_{\beta_{i}^{0}}(z)}, \quad i=1, \ldots, m
$$

locally uniformly in $\mathbb{C}^{I} \backslash\left\{\beta_{i}^{0}\right\}$ where $z=J^{-1}(x), \beta_{i}^{0}=J^{-1}\left(\alpha_{i}^{0}\right)$ and $\alpha_{0}^{0}=\alpha_{m}^{0}$. This is a meromorphic function which has a single pole at $\alpha_{i}^{0}$.

Proof. Use lemma 6.3 and the proof of theorem 6.1 to obtain (9). This is a meromorphic function since $S_{w}(z)$ is analytic and does not vanish in $\mathbb{D}$.

Finally we come to the most general theorem in this section, where the only assumption about the poles is that they stay away from the boundary of the interval $[-1,1]$. The following theorem is again a simple consequence of theorems 4.1 and 5.1 and we omit the proof. 
Theorem 6.5. Assume the sequence $A=\left\{\alpha_{1}, \alpha_{2}, \ldots\right\} \subset \mathbb{R}^{I}$ is bounded away from $I$ and the measure $\mu$ satisfies the conditions of theorem 6.1. If $\left\{\varphi_{n}\right\}$ are the orthonormal rational functions on I associated with $A$ and $\mu$, then the sequence

$$
\left\{\frac{\varphi_{n+1}(x)}{\varphi_{n}(x)}\right\}, \quad n=0,1, \ldots
$$

is locally uniformly bounded in $\mathbb{C}^{I} \backslash A$.

The relation between orthogonal rational functions on the unit circle and those on the interval can of course be used to find other asymptotic formulas as well. We give one example in the following theorem.

Theorem 6.6. Under the assumptions of theorem 6.4, we have for $x \in \mathbb{C}^{I}$, $i=1, \ldots, m$ and $n \rightarrow \infty$,

$$
\varphi_{n m+i}(x) \approx(2 \pi)^{-\frac{1}{2}} \prod_{k=1}^{n m+i}\left(\frac{1-\alpha_{k} x}{x-\alpha_{k}}+\frac{\sqrt{\left(x^{2}-1\right)\left(\alpha_{k}^{2}-1\right)}}{x-\alpha_{k}}\right) S_{\beta_{i}^{0}}\left(J^{-1}(x)\right)
$$

where the square roots are chosen so that each factor in the product has modulus less than one and $\beta_{i}^{0}=J^{-1}\left(\alpha_{i}^{0}\right)$. This formula holds in the sense that the ratio tends locally uniformly to 1 in $\mathbb{C}^{I}$. In particular we have

$$
\lim _{n \rightarrow \infty} \varphi_{n}(x)=\infty, \quad \text { pointwise for } x \in \mathbb{C}^{I} .
$$

which also holds under the less restrictive assumptions of theorem 6.5.

Proof. As in the proof of theorem $6.1, \varphi_{n}(x)$ can be written

$$
\varphi_{n}(x)=\frac{1}{B_{n}(z)}(2 \pi)^{-\frac{1}{2}}\left\{1+\frac{\tilde{\phi}_{2 n}\left(\beta_{n}\right)}{\tilde{\kappa}_{2 n}}\right\}^{-\frac{1}{2}}\left\{\tilde{\phi}_{2 n}(z)+\tilde{\phi}_{2 n}^{*}(z)\right\} .
$$

The result is immediate from theorem 4.1, corollary 6.2 and the fact that the Blaschke product $B_{n}(z)$ diverges to zero for $z \in \mathbb{D}$. 


\section{Asymptotics for $E_{n}$ and $B_{n}$}

In this section we wish to derive asymptotic formulas for the recurrence coefficients $E_{n}$ and $B_{n}$. First we note that explicit formulas for the coefficients in terms of the orthogonal functions $\varphi_{n}$ are given by

$$
\begin{aligned}
& E_{n}=\lim _{x \rightarrow \alpha_{n-1}} \frac{\varphi_{n}(x)}{\varphi_{n-1}(x) Z_{n}(x)}, \\
& B_{n}=\lim _{x \rightarrow \alpha_{n-2}}\left(\frac{\varphi_{n}(x)}{\varphi_{n-1}(x)} \frac{Z_{n-1}(x)}{Z_{n}(x)}-E_{n} Z_{n-1}(x)\right) .
\end{aligned}
$$

These formulas are readily obtained from the recurrence formula (1). Now we can use our main theorem 6.1 to find the asymptotic formulas for $E_{n}$ and $B_{n}$, as shown in the following theorem.

Theorem 7.1. Under the assumptions of theorem 6.1, the following relations hold,

$$
\begin{aligned}
& \lim _{n \rightarrow \infty} E_{n}=2\left(1-1 / \alpha^{2}\right), \\
& \lim _{n \rightarrow \infty} B_{n}=-2 / \alpha .
\end{aligned}
$$

Proof. Put as usual $x=J(z)$ and $\alpha_{k}=J\left(\beta_{k}\right)$ then the product $Z_{n}(x) \zeta_{n}(z)$ has no zero for $x \in \mathbb{C}^{I}$ or equivalently, $z \in \mathbb{D}$. The zero $z=\beta_{n}$ in $\zeta_{n}$ is cancelled against the pole $x=\alpha_{n}$ in $Z_{n}$ and the zero $x=0$ in $Z_{n}$ is not in $\mathbb{C}^{I}$. Therefore, using theorem 6.1 ,

$$
\lim _{n \rightarrow \infty} \frac{\varphi_{n}(x)}{\varphi_{n-1}(x) Z_{n}(x)}=\frac{F_{\alpha}(x)}{Z_{\alpha}(x)}
$$

locally uniformly in $\mathbb{C}^{I}$, where $Z_{\alpha}(x)=-\alpha x /(x-\alpha)$.

From theorem 3.1 and the previous remark it follows that $\varphi_{n} /\left(\varphi_{n-1} Z_{n}\right)$ and $F_{\alpha} / Z_{\alpha}$ are continuous in $\mathbb{C}^{I}$. Because of the uniform convergence we then have from formula (10)

$$
\lim _{n \rightarrow \infty} E_{n}=\lim _{x \rightarrow \alpha} \frac{F_{\alpha}(x)}{Z_{\alpha}(x)} .
$$

The result then follows from the Laurent series expansion (7) if $\alpha$ is finite, or from formula (8) otherwise. 
Using a similar argument we obtain the formula for $B_{n}$.

Again we note the correspondence with the polynomial case. If $\alpha$ equals infinity, the recursion coefficients will behave asymptotically as the recursion coefficients in the well known recurrence formula for orthogonal polynomials on $I$, see e.g. [9, p.310] for the case of an absolutely continuous measure satisfying Szego's condition, and [6, p.212] for the general situation.

In the case of an asymptotically periodic pole sequence with $m>1$ the expressions are a bit more involved, but the argument is the same and we give the theorem without proof.

Theorem 7.2. Under the assumptions of theorem 6.4, the following relations hold for $i=1, \ldots, m$,

$$
\begin{aligned}
\lim _{n \rightarrow \infty} E_{n m+i} & =\lim _{x \rightarrow \alpha_{i-1}^{0}} \frac{S_{\beta_{i}^{0}, \beta_{i-1}^{0}}\left(J^{-1}(x)\right)}{\zeta_{\beta_{i}^{0}}\left(J^{-1}(x)\right) Z_{\alpha_{i}^{0}}(x)}, \\
\lim _{n \rightarrow \infty} B_{n m+i} & =\lim _{x \rightarrow \alpha_{i-2}^{0}}\left(\frac{S_{\beta_{i}^{0}, \beta_{i-1}^{0}}\left(J^{-1}(x)\right)}{\zeta_{\beta_{i}^{0}}\left(J^{-1}(x)\right) Z_{\alpha_{i}^{0}}(x)}-\lim _{n \rightarrow \infty} E_{n m+i}\right) Z_{\alpha_{i-1}^{0}}(x) .
\end{aligned}
$$

These formulas can be made explicit using lemma 6.3 and a lot of algebra. As an example, we just give the result for the coefficient $E_{n}$ :

$$
\begin{aligned}
E_{n m+i} \rightarrow \frac{1}{2} \frac{\left(\sqrt{\left(\alpha_{i}^{0}\right)^{2}-1}+\sqrt{\left(\alpha_{i-1}^{0}\right)^{2}-1}\right)^{2}-\left(\alpha_{i}^{0}-\alpha_{i-1}^{0}\right)^{2}}{\alpha_{i}^{0} \alpha_{i-1}^{0}} \\
\quad\left\{\frac{1-\left(J^{-1}\left(\alpha_{i}^{0}\right)\right)^{2}}{1-\left(J^{-1}\left(\alpha_{i-1}^{0}\right)\right)^{2}}\right\}^{\frac{1}{2}} \exp \left\{-\frac{J^{-1}\left(\alpha_{i-1}^{0}\right)}{\pi} \int_{0}^{2 \pi} \frac{\log \left|\frac{e^{\mathrm{i} \theta}-J^{-1}\left(\alpha_{i}^{0}\right)}{e^{\mathrm{i} \theta}-J^{-1}\left(\alpha_{i-1}^{0}\right)}\right|}{e^{\mathrm{i} \theta}-J^{-1}\left(\alpha_{i-1}^{0}\right)} d \theta\right\} .
\end{aligned}
$$

The last theorem we present corresponds to the general situation which only requires that the poles stay away from $I$, as in theorem 6.5.

Theorem 7.3. Under the assumptions of theorem 6.5, the sequences $\left\{E_{n}\right\}$ and $\left\{B_{n}\right\}$ are bounded.

Proof. The sequence $\varphi_{n}(x) /\left(\varphi_{n-1}(x) Z_{n}(x)\right)$ is locally uniformly bounded in $\mathbb{C}^{I}$ and each of its elements is a continuous function in $\mathbb{C}^{I}$. Therefore the sequence $\left\{E_{n}\right\}$ is bounded. A similar argument holds for $\left\{B_{n}\right\}$. 


\section{Conclusion}

In this paper we studied the asymptotic behavior of the ratio of orthogonal rational functions on the interval $[-1,1]$ with real poles outside this interval. We obtained convergence results if the measure satisfies a Szegő condition and under certain assumptions on the location of the poles. It remains to be investigated if these results also hold for arbitrary positive measures which satisfy $\mu^{\prime}>0$ a.e. (as in the polynomial case).

\section{References}

[1] A. Bultheel, P. González-Vera, E. Hendriksen, and O. Njåstad. Orthogonal Rational Functions, volume 5 of Cambridge Monographs on Applied and Computational Mathematics. Cambridge University Press, Cambridge, 1999.

[2] J. Van Deun and A. Bultheel. Orthogonal rational functions and quadrature on an interval. J. Comput. Appl. Math., 2001. submitted.

[3] G. Freud. Orthogonal Polynomials. Pergamon Press, Oxford, New York, 1971.

[4] G. L. López. On the asymptotics of the ratio of orthogonal polynomials and convergence of multipoint padé approximants. Math. USSR Sbornik, 56:207-219, 1985.

[5] G. L. López. Asymptotics of polynomials orthogonal with respect to varying measures. Constr. Approx., 5:199-219, 1989.

[6] E. A. Rakhmanov. On the asymptotics of the ratio of orthogonal polynomials. Math. USSR Sbornik, 32:199-213, 1977.

[7] E. A. Rakhmanov. On the asymptotics of the ratio of orthogonal polynomials. II. Math. USSR Sbornik, 46:105-117, 1983.

[8] W. Rudin. Real and Complex Analysis. McGraw-Hill, New York, 1987. 3rd ed.

[9] G. Szegö. Orthogonal Polynomials, volume 33 of Am. Math. Soc. Colloq. Publ. Am. Math. Soc., Providence, RI, 1967. 
[10] P. Van gucht and A. Bultheel. A relation between orthogonal rational functions on the unit circle and the interval $[-1,1]$. Comm. Anal. Th. Continued Fractions, 8:170-182, 2000. 